\section{Evaluación de la enfermedad de Alzheimer en etapa temprana: biomarcadores y pruebas neuropsicológicas}

\author{
RENZO LANFRANCO G. ${ }^{1,2, a}$, PAULA MANRÍQUEZ-NAVARRO ${ }^{2, b}$, \\ LEYLA AVELLO G. ${ }^{3, c}$, ANDRÉS CANALES-JOHNSON ${ }^{1, \mathrm{~d}}$
}

\section{Early evaluation of Alzheimer's disease: biomarkers and neuropsychological tests}

\begin{abstract}
Alzheimer disease is a neurodegenerative condition that affects cognitive, behavioral and global functioning of patients. Currently and due to the lack of conclusive biological testing, Alzheimer's disease diagnosis is based primarily on clinical criteria. Since its early diagnosis allows clinical interventions when neurological damage is relatively mild, the development of early detection tools has become a major topic of interest. In this article, we review the main neurobiological and neuropsychological features of Alzheimer's disease, analyzing the use of biomarkers and neuropsychological testing for early detection.
\end{abstract}

(Rev Med Chile 2012; 140: 1191-1200).

Key words: Alzheimer disease; Biological markers; Neurobiology; Neuropsychology.

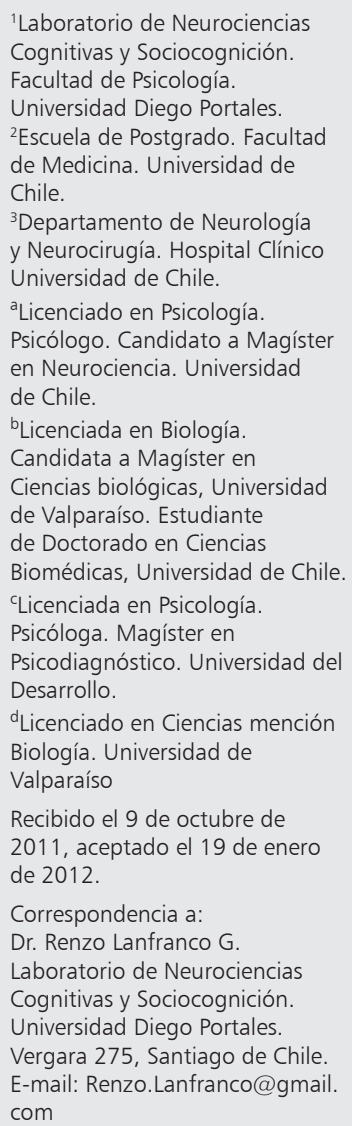

L a enfermedad de Alzheimer (EA) es una afección neurodegenerativa caracterizada por deterioro cognitivo, conductual y del funcionamiento global, de inicio insidioso y curso progresivo, de aparición en edad adulta, primordialmente en la vejez ${ }^{1}$. Su prevalencia aumenta con la edad, estimándose unos valores entre $0,6 \%$ y 1\% en el rango de los 65-69 años y cercano al $30 \%$ en edades iguales o superiores a los 90 años $^{2}$. La EA de inicio temprano es poco frecuente (antes de los 60 años de edad), y sólo representa entre el $1 \%$ y el $3 \%$ de los casos. Debido a que no existen pruebas biológicas definitivas para su detección, el diagnóstico se basa fundamentalmente en la aplicación de criterios clínicos ${ }^{3}$.

La detección de la EA en etapas tempranas se ha convertido en uno de los principales focos de investigación en enfermedades neurodegenerativas. Su detección y diagnóstico temprano permite realizar ensayos clínicos e intervenciones eficaces cuando el daño neurológico es aún relativamente leve.

Esta revisión pretende exponer algunas generalidades clínicas de la EA, tanto respecto a sus criterios diagnósticos como a su curso y perfil neuropsicológico, además de sus correlatos neurobiológicos característicos, para luego señalar estrategias para el diagnóstico temprano en la EA. Incluimos la exposición de biomarcadores y pruebas neuropsicológicas utilizadas actualmente.

\section{Aspectos clínicos de la EA}

\section{Criterios diagnósticos}

Los criterios para diagnosticar EA se basan principalmente en síntomas del orden cognitivo, conductual y social, a través de una evaluación neurocognitiva del paciente y de entrevista a familiares. En el caso de los criterios expuestos en el DSM IV-tr, entre otros, para realizar el diagnóstico 
es necesario que el paciente presente déficit en la memoria y, en segundo lugar, deterioro en al menos una de las siguientes funciones: lenguaje, praxias, gnosis y funcionamiento ejecutivo, causando un deterioro significativo en su actividad social y laboral en comparación al estadio premórbido. Este deterioro debe ser gradual y continuo, y no deberse a otras enfermedades neurológicas, sistémicas o por consumo de sustancias ${ }^{4}$.

Actualmente, se han consensuado nuevas entidades nosológicas con la intención de clasificar a los pacientes con alteraciones cognitivas leves (Deterioro Cognitivo Leve o DCL) que potencialmente pudieran evolucionar a una demencia ${ }^{5,6}$. Sin embargo, se ha descrito que el DCL no necesariamente llevaría a una EA u otra demencia; algunos casos vuelven a un estado cognitivo normal ${ }^{7}$.

\section{Curso neuropsicológico}

Típicamente la EA comienza insidiosamente con pérdida de la memoria episódica, dificultades para aprender y retener nueva información, pérdida gradual de la orientación temporal y de referencias contextuales a fechas, implicando alteraciones de memoria autobiográfica ${ }^{8}$. Ello correlaciona con alteraciones relacionadas con la anatomofisiología de la corteza entorrinal y el hipocampo. Estas características tipifican su fase prodrómica. Sin embargo, la memoria de hechos remotos suele conservarse y, en ocasiones, potenciarse. Por otro lado, las memorias procedural y semántica suelen conservarse en las fases iniciales ${ }^{3}$. La alteración en memoria episódica es tanto para el material visual como verbal ${ }^{9}$, dato relevante a la hora de realizar un diagnóstico diferencial con demencia frontotemporal, la cual suele presentar un deterioro más hemisférico, implicando diferencialmente alteraciones episódicas visuales o verbales ${ }^{10}$.

En fases medianamente avanzadas, cuando las alteraciones anatomopatológicas progresan hacia zonas corticales temporales laterales, suelen aparecer alteraciones en lenguaje y memoria semántica ${ }^{11}$, siendo frecuente la anomia, además de alteraciones en comprensión del lenguaje y fluencia verbal. La escritura suele conservarse hasta etapas más avanzadas ${ }^{10}$.

En fases más avanzadas, cuando el deterioro alcanza regiones temporoparietales, suelen aparecer apraxias (e.g. constructiva e ideomotora en fases tempranas; y del vestir, en tardías), agnosias (e.g. visuales) y alteraciones en la lectoescritura ${ }^{3}$. Las alteraciones en funciones cognitivas frontales incluyen anosognosia, alteraciones de la conducta social y control de impulsos. Además, suelen presentarse afecciones en función ejecutiva, como inflexibilidad cognitiva, incapacidad para planificar y abstraer, etc ${ }^{12}$.

En fases más tardías, suele verse signos de deterioro subcortical como alteraciones de la marcha (e.g. marcha parkinsoniana) y deglución. El paciente suele quedar postrado, con pérdida del juicio y alteraciones de conciencia, encontrando la muerte como desenlace de la enfermedad.

\section{Correlatos neurobiológicos de la EA}

\section{Descripción molecular}

En la EA, a nivel de los tejidos nerviosos, se puede observar depósitos extracelulares de péptido beta amiloide $(\mathrm{A} \beta)$, llamadas placas amiloideas, $y$ ovillos neurofibrilares de proteína tau fosforilada, acompañada de degeneración sináptica y neuronal, especialmente en corteza e hipocampo.

La causa de la muerte neuronal aún no se ha determinado, pero se encuentran ampliamente estudiados los efectos de toxicidad de $\mathrm{A} \beta$ sobre cultivos celulares y su importancia en el desarrollo de la $\mathrm{EA}^{13}$. $\mathrm{A} \beta$ es producto de la proteína precursora de amiloide (APP) que es clivada por proteasas (secretasas $\alpha, \beta, \gamma$ ); el sitio de clivaje determinará el origen de varias isoformas de $\mathrm{A} \beta$ que tienen entre 36 y 43 aminoácidos. Investigaciones recientes han asociado la isoforma $A \beta_{42}$ en la formación de placas amiloideas ${ }^{14}$. Si bien son numerosos los mecanismos que definen a $A \beta$ como el principal responsable de la muerte neuronal (activación de microglias, aumento de citoquinas neurotóxicas, daño oxidativo, etc.), aún no es posible determinar satisfactoriamente la mecánica molecular necesaria y suficiente de la $\mathrm{EA}^{15}$.

Sobre el desarrollo de la enfermedad, estudios recientes sugieren que, a diferencia de lo que se creía antiguamente, las placas amiloideas difícilmente serían la causa, por su aparición tardía en el curso de la EA. Por otro lado, se ha demostrado una alta correlación entre la concentración de oligómeros solubles a nivel cerebral y alteraciones neurológicas ${ }^{16}$. También se ha sugerido que la formación de canales de oligómeros de amiloide (canales amiloídeos) serían los responsables de la fisiopatología de la EA. Estos canales se caracteri- 
zan por ser permeables a calcio y, al encontrarse en la membrana, causarían un cambio en la homeostasis del mismo ion, gatillando apoptosis ${ }^{17}$.

\section{Descripción histopatológica}

En tejidos obtenidos post mortem se han identificado las zonas con alteraciones más graves: hipocampo, subiculum, amígdala y áreas de asociación neocortical. En hipocampo y corteza temporal es común encontrar un patrón topográfico asociado a las etapas histopatológicas tempranas y tardías de la $\mathrm{EA}^{18}$. La acumulación de filamentos insolubles caracterizados por su conformación de $\beta$-plegada (A $\beta$ mal plegada) definen dos tipos principales de lesiones: las placas de amiloide y los ovillos neurofibrilares. El núcleo basal de Meynert (i.e. área innominata) suele presentar pérdida neuronal, formación de estos ovillos y ausencia de placas de $A \beta^{19}$. Otra característica es la presencia de hilos de neurópilo, depósitos de amiloide (vasos sanguíneos cerebrales y meníngeos), degeneración gránulo-vacuolar en las células piramidales del hipocampo, cuerpos de Hirano, gliosis reactiva, aumento de las células de la microglia y alteraciones pseudoespongiformes ${ }^{20}$.

Recientemente se ha propuesto que los depósitos amorfos amiloideos vinculados con canales amiloideos serían los principales causantes de la fisiopatología de la $\mathrm{EA}^{20}$.

\section{Diagnóstico y detección temprana de la EA}

\section{Biomarcadores}

Un biomarcador para la EA debe ser una característica biológica medible de la actividad normal o patológica del cerebro, que pueda emplearse para diagnosticar el riesgo, gravedad o progresión de la enfermedad, además de ser útil para monitorizar la respuesta a medidas terapéuticas ${ }^{21}$. En la actualidad, se investigan distintos tipos de biomarcadores, pudiéndose clasificar según su fuente de obtención ${ }^{22,23}$.

En neuroimagen se han utilizado técnicas como resonancia nuclear magnética (MRI), tomografía por emisión de positrones (PET) y morfometría basada en voxel (VBM), permitiendo distinguir cambios estructurales y metabólicos asociados al curso de la EA. Con VBM se han detectado disminuciones tempranas de volumen $y$ densidad en materia gris ${ }^{24}$, y pérdida de materia gris en los lóbulos frontal, temporal inferior y medial ${ }^{25}$. También, se han medido alteraciones metabólicas con PET, permitiendo discriminar in vivo entre EA y sanos ${ }^{26}$. Se usan contrastes que se ligan a placas amiloídeas, ovillos neurofibrilares, microglia activada y astrocitos reactivos, como $\mathrm{PiB}$, encontrándose retención aumentada en cortezas frontales, parietales, temporales, occipitales y cuerpo estriado ${ }^{27}$.

El líquido cefalorraquídeo (LCR) mantiene contacto directo con el espacio subaracnoídeo y el sistema ventricular ${ }^{23}$, obteniéndose con punción lumbar. Tau y A $\beta$ son los más estudiados, por su rol en las placas amiloídeas y ovillos neurofibrilares ${ }^{28}$. La isoforma $\mathrm{A} \beta_{42}$, participante en la formación de oligómeros y fibras que se acumulan en placas extracelulares, se ve disminuido en LCR en EA ${ }^{29-}$ ${ }^{31}$. Además, presentan alta correlación entre su disminución y el aumento de la cantidad de placas amiloídeas medidas por el índice $\mathrm{PET}-\mathrm{PiB}^{32} . \mathrm{A} \beta_{40}$ se ha estudiado sin lograr distinguir EA de sanos. Sin embargo, una proporción disminuida en $\mathrm{A} \beta_{42} / \mathrm{A} \beta_{40}$ ha demostrado ser mejor biomarcador de EA que $\mathrm{A} \beta_{42}$ aislada $^{33}$. Algo similar ocurre con $\mathrm{A} \beta_{42} / \mathrm{A} \beta_{38}{ }^{34}$. Otra proteína de interés en LCR es tau. En EA, tau se hiperfosforila, disociándose de los microtúbulos, polimerizándose subsecuentemente en filamentos helicoidales insolubles. Estos filamentos contribuyen a la formación de los ovillos neurofibrilares ${ }^{35}$. Niveles elevados de tau total ( $\mathrm{t}$-tau) y tau fosforilada ( $\mathrm{f}$-tau) correlacionan fuertemente con EA. Tau como biomarcador ha demostrado ser muy sensible ${ }^{36}$. Pero f-tau es más sensible y específico ${ }^{37,38}$. Tau ( $t$-tau y f-tau en treonina 231) también han demostrado servir para identificar a sujetos con DCL que desarrollarán una EA; f-tau en treonina 231 también distingue entre EA y otras neuropatologías ${ }^{39}$.

Existen biomarcadores genéticos de riesgo, cuya presencia en el genoma no asegura la manifestación de EA. Encontramos mutaciones en el alelo $\varepsilon 4$ de la apolipoproteína E $(\mathrm{APOE})^{40}$ y las mutaciones PS1 en el cromosoma 14, PS2 en el cromosoma 1 y PPA en el cromosoma 21 (vinculado a EA familiar de inicio temprano). El primero es frecuente en la población, pero su correlación con EA es baja, mientras que con el segundo se da lo contrario ${ }^{41}$.

Otras sustancias relevantes en LCR son los isoprostanos F2 (IPF2), elevados en pacientes con EA, correlacionando con t-tau y $\mathrm{A}_{42}{ }^{42}$. Pacientes con DCL con alta concentración de IPF2A 
tienen alta probabilidad de evolucionar a $\mathrm{EA}^{43,44}$. También se han explorado proteínas sinápticas (e.g. sensores de calcio), encontrándose niveles significativamente elevados en LCR, demostrando sensibilidad y especificidad comparables a t-tau y $\mathrm{f}$-tau ${ }^{45}$. Combinaciones con $A \beta_{42}$, f-tau y VLP-1 (Visinin-like-protein-1) aumentan la precisión diagnóstica.

Otro enfoque es la combinación de marcadores. Altas concentraciones de t-tau junto a bajas concentraciones de $A \beta_{42}$ pueden detectar la EA con alta sensibilidad y especificidad ${ }^{46}$. Lo mismo ocurre para altas proporciones en concentración de $\mathrm{f}$-tau/ $\mathrm{A} \beta_{42}{ }^{47}$, teniendo más potencial como biomarcador de diagnóstico que de progresión ${ }^{48}$. Se identificaron 18 proteínas en plasma, conformando un algoritmo para distinguir entre EA y sanos, con una precisión de $90 \%$, prediciendo qué pacientes con DCL progresarían a $\mathrm{EA}^{49}$. También se construyó un algoritmo usando diferencias de concentración proteica en suero de 121 proteínas vinculadas a inflamación, remodelamiento citoesquelético y señalización celular, factores de crecimiento, hormonas y otras, con edad, sexo, años de escolaridad y genotipo $\mathrm{APOE}^{50}$.

Otra estrategia corresponde a estudios de conectividad funcional de la default-mode network (DMN). La DMN corresponde a regiones del cerebro cuya actividad aumenta en estados de reposo ${ }^{51}$, de actividad mental espontánea y de evocación de memoria episódica y autobiográfica involuntaria ${ }^{52}$, y disminuye en tareas dirigidas atencionalmente ${ }^{53}$. Estas regiones se hallan funcionalmente conectadas ${ }^{54}$. Se ha descubierto que la EA implica una disrupción en nodos específicos de la $\mathrm{DMN}^{55}$, especialmente en la corteza cingulada posterior (CCP). Esta disrupción en CCP decrecería gradualmente en relación directa a la severidad de la EA. Damoiseaux et al (2011) compararon la conectividad de cuatro redes vinculadas a estados de descanso en la progresión de la enfermedad. Descubrieron que la DMN posterior comienza a perder conectividad en etapas tempranas, mientras DMN ventral y anterior aumentan su conectividad $^{56}$. Otros estudios se han centrado en los volúmenes de sustancia gris de nodos específicos de la $\mathrm{DMN}$, encontrando disminución en la conectividad de hipocampo, lóbulo temporal medial y CCP en DCL de tipo amnésico ${ }^{57}$. Gili et al (2011) han descubierto que la desconexión funcional de DMN precedería esta atrofia de sustancia gris en $\mathrm{PCC}^{58}$.
Petrella et al (2011) usando índice Goodness-ForFit en un estudio sobre volúmen de materia gris en nodos de la DMN, incluyendo datos como edad, puntuación en CDR (Clinical Dementia Rating), años de escolaridad y MMSE (Mini Mental Score Examination), lograron predecir qué pacientes con DCL amnésico progresarían a EA y cuáles no ${ }^{59}$.

La evidencia empírica indica que los nodos de la DMN se hallarían especialmente vulnerables a la precipitación de $A \beta^{60}$, especialmente DMN posterior, corteza prefrontal medial, hipocampo, precuneo y $\mathrm{CCP}^{61}$, entre otras. Otros estudios sugieren que la misma actividad de la DMN podría acelerar el depósito de $A \beta$ en sus nodos ${ }^{62}$.

En la Tabla 1 se exponen algunos biomarcadores y sus respectivos índices de sensibilidad y especificidad. Se distingue entre biomarcadores simples y complejos, según si es compuesto único o involucra un algoritmo entre varios compuestos.

\section{Exploración neuropsicológica}

El objetivo principal de la exploración neuropsicológica en EA consiste en demostrar la existencia de trastornos cognitivos y determinar sus características e intensidad. Esta exploración persigue identificar capacidades preservadas y afectadas para bosquejar un perfil clínico del paciente ${ }^{63}$. Es importante distinguir entre las distintas afecciones que pueden encontrarse en el paciente con EA; no todas sus manifestaciones serán neuropsicológicas. De hecho, es posible que las diferencias en el orden de los trastornos no neuropsicológicos (e.g. afecciones psiquiátricas) sean más esclarecedoras al momento de realizar un diagnóstico diferencial entre demencias. Sin embargo, en lo que respecta a síntomas neuropsicológicos, se encontrará con frecuencia apraxia, agnosia, acalculia, alexia, agrafia y síndromes focales de afasia, entre otros ${ }^{64}$.

Peña-Casanova (2006) define que el proceso de exploración neuropsicológica debe ser modular, reconociendo y cuantificando los trastornos del paciente. Como no existe una batería neuropsicológica útil para todo tipo de necesidades y de pacientes, la flexibilidad es fundamental ${ }^{65}$ :

\section{Exploración básica: entrevistas estructuradas y pruebas breves de cribado}

Además de los datos obtenidos mediante entrevista clínica, anamnesis y examen neurológico, 
Tabla 1. Sensibilidad y especificidad en biomarcadores de EA en fase temprana

\begin{tabular}{|c|c|c|c|}
\hline & & Sensibilidad & Especificidad \\
\hline \multirow{7}{*}{$\begin{array}{l}\text { Biomarcadores } \\
\text { simples }\end{array}$} & Presencia de APOE $\varepsilon 4$ & $65 \%$ & $68 \%$ \\
\hline & Atrofia neuronal mediante VBM & $94 \%$ & $89 \%$ \\
\hline & Concentración de $A \beta_{1-42}$ en LCR & $100 \%$ & $94 \%$ \\
\hline & Concentración de Tau Total en LCR & $84 \%$ & $94 \%$ \\
\hline & Concentración de Tau Fosforilada en LCR & $88 \%$ & $97 \%$ \\
\hline & Proporción entre Tau Fosforilada y $A \beta_{42}$ & $88 \%$ & $89 \%$ \\
\hline & Conectividad funcional en DMN con índice GOF mediante fMRI & $85 \%$ & $77 \%$ \\
\hline \multirow[t]{3}{*}{$\begin{array}{l}\text { Biomarcadores } \\
\text { complejos }\end{array}$} & $\begin{array}{l}\text { Niveles bajos de } A \beta_{1-42} \text {, niveles altos de proteína tau total y tau } \\
\text { fosforilada }_{181 P} \text { en LCR. }\end{array}$ & $94 \%$ & - \\
\hline & $\begin{array}{l}\text { Concentración de proteínas en suero asociadas a inflamación, } \\
\text { remodelación citoesquelética y señalización celular, además de } \\
\text { factores de crecimiento, hormonas, etc., en combinación con } \\
\text { factores edad, sexo, años de educación y genotipo ApoE. }\end{array}$ & $94 \%$ & $84 \%$ \\
\hline & Combinación en LCR de $A \beta_{1-42}$ y tau total & $88 \%$ & $89 \%$ \\
\hline
\end{tabular}

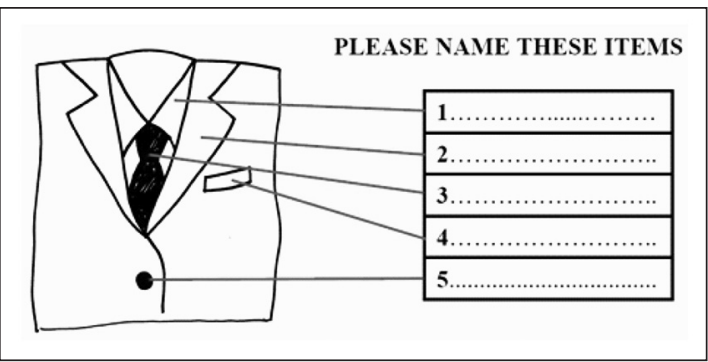

Figura 1. Nominación en el TYM Test: El TYM Test es una prueba de cribado autoadministrada. Estudios recientes señalan que es capaz de detectar el $93 \%$ de los pacientes con EA, siendo mucho más sensible que el MMSE, el cual sólo detecta a $52 \%$ de los pacientes con la misma enfermedad. Reproducción de la Figura 1 ha sido autorizada por el autor. Ver referencia 71). El Test se encuentra en el sitio http://www. tymtest.com, para su descarga gratuita.

existen entrevistas estructuradas que tienen como objetivo recabar datos específicos sobre las funciones mentales implicadas en la enfermedad, como The Mental Status Examination in Neurology ${ }^{66} \mathrm{y}$ The Psychiatric Mental Status Examination ${ }^{67}$.

Las pruebas breves de cribado tienen como objetivo evaluar de manera rápida y simple los distintos ámbitos cognitivos. Se obtiene un índice del estado cognitivo que a partir de puntos de corte sugiere presencia o ausencia de patología. Entre ellos encontramos el Mini Mental State Examination de Folstein $(1975)^{68}$, el Memory Impairment Screen ${ }^{69}$, Clock Drawing Test ${ }^{70}$ y el TYM Test de Brown et al.
$(2009)^{71}$. En la Figura 1 se expone el ejercicio de nominación de la prueba autoaplicada TYM test, la cual actualmente es una de las pruebas breves de cribado con mayor sensibilidad para detectar EA.

\section{Exploración general breve-intermedia}

En esta etapa se introducen pruebas neuropsicológicas específicas para EA. Todas ya poseen estandarización y evalúan múltiples funciones: Alzheimer's Disease Assessment Scale (ADAS) ${ }^{72}$, Cambridge Index of Mental Disorder in the Elderly (CAMDEX) $)^{73}$, Hierarchic Dementia Rating Scale $(\mathrm{HDR})^{74}$ y The Montreal Cognitive Assessment $(\mathrm{MoCA})^{75}$, entre otras. Evalúan reconocimiento, memoria, praxis constructiva, intensidad del deterioro, lenguaje, gnosis, conducta motora, signos frontales, etc.

\section{Exploración general detallada: selección de prueba}

Aquí se seleccionan las pruebas más apropiadas para la evaluación acuciosa, hipotetizando una posible etiología. Éstas suelen clasificarse dependiendo de las funciones que exploran. En la Tabla 2 se señalan algunas de las pruebas neuropsicológicas más usadas para evaluar $\mathrm{EA}^{76}$.

\section{Exploración idiográfica}

Corresponde a una exploración dirigida en función de la hipótesis sobre la ubicación funcional del trastorno neuropsicológico ${ }^{65}$. Se comienza 
a partir del modelo sobre la función normal para elegir pruebas que permitan definir el trastorno del individuo evaluado. Se pueden aplicar pruebas existentes o crear pruebas específicas para el individuo en cuestión. Esta fase es imprescindible para estudiar casos únicos. A través de hipótesis funcionales se pretende explicar el patrón clínico observado.

Para la detección temprana de la EA es necesario utilizar una amplia gama de pruebas, con el objetivo de pesquisar las funciones mentales que suelen deteriorarse tempranamente, como: memoria episódica y semántica,

Tabla 2. Pruebas neuropsicológicas según función evaluada en EA

\begin{tabular}{|ll|}
\hline Funciones cognitivas & Pruebas neuropsicológicas \\
Funciones ejecutivas y \\
atención-concentración & $\begin{array}{l}\text { Test de Stroop } \\
\text { Torre de Londres } \\
\text { Wisconsin Card Sorting Test } \\
\text { Trail Making Test }\end{array}$ \\
Memoria episódica verbal & $\begin{array}{l}\text { Rey Auditory Verbal Learning Test } \\
\text { Wechsler Memory Scale III (WMS) } \\
\text { California Verbal Learning Test II }\end{array}$ \\
Memoria episódica visual & $\begin{array}{l}\text { Figura Compleja de Rey-Osterrieth } \\
\text { Benton Visual Retention Test }\end{array}$ \\
Memoria de trabajo & $\begin{array}{l}\text { Subtest de Dígitos (WAIS) } \\
\text { Lenguaje }\end{array}$ \\
$\begin{array}{l}\text { Boston Naming Test (Nominación) } \\
\text { Token Test (Comprensión y percepción) } \\
\text { Test de Fluencia Verbal }\end{array}$ \\
$\begin{array}{ll}\text { Habilidades visuoespa- } \\
\text { ciales }\end{array}$ & $\begin{array}{l}\text { Figura Compleja de Rey-Osterrieth } \\
\text { Test de Bender BIP } \\
\text { Subtest de Cubos (WAIS) } \\
\text { Subtest de Rompecabezas (WAIS) }\end{array}$ \\
\hline $\begin{array}{l}\text { Razonamiento lógico y } \\
\text { abstracción }\end{array}$ & $\begin{array}{l}\text { Subtest de Comprensión (WAIS) } \\
\text { Subtest de Semejanzas (WAIS) }\end{array}$ \\
\hline
\end{tabular}

atención, lenguaje, funciones ejecutivas, visuoespaciales y visuoperceptivas ${ }^{65}$.

En la evaluación de memoria episódica, estudios sugieren que medir la capacidad de aprender nueva información es uno de los mejores indicadores para diferenciar entre personas sanas y con $\mathrm{EA}^{65}$. Se evalúa la tasa rápida de olvido, pues la evocación mnésica en EA es deficitaria ${ }^{77}$. La evaluación debe enfocarse en codificación y almacenamiento de información, capacidad de aprendizaje, evocación libre y reconocimiento de material previamente presentado ${ }^{76}$. El déficit en memoria semántica se evalúa en tareas de evocación categorial o denominación. Las expresiones vacías y circunloquios son comunes en $\mathrm{EA}^{78}$. En la Tabla 3 se señalan algunas pruebas neuropsicológicas para evaluar memoria, con sus respectivos índices de confiabilidad.

Las funciones ejecutivas y atenciónconcentración suelen verse tempranamente alteradas ante tareas de resolución de problemas, abstracción y razonamiento lógico ${ }^{65}$. Además, suele encontrarse rigidez cognitiva, planificación conductual pobre y control pobre en la supervisión atencional ${ }^{76}$.

Dentro de las funciones lingüísticas, suele presentarse anomia en fases tempranas a intermedias en el curso. Se presenta disminución en la espontaneidad verbal y dificultad en la fluidez ${ }^{76}$.

El deterioro de las funciones visuoperceptivas y visuoconstructivas aparece tras

Tabla 3. Pruebas neuropsicológicas para evaluar memoria en EA y confiabilidad

\begin{tabular}{|llll|}
\hline & & Confiabilidad \\
Memoria episódica verbal & Rey Auditory Verbal Learning Test & Interna & Test-retest \\
& Wechsler Memory Scale III (WMS) &, 90 &, 70 \\
& California Verbal Learning Test II & Entre, 81 y, 87, 82 & Entre, 80 y, 84 \\
Memoria episódica visual & Figura Compleja de Rey-Osterrieth (Copia y Recuerdo) &, 60 y, 80 & Entre, 76 y, 89 \\
& Benton Visual Retention Test (Forma A) &, 79 &, 85 \\
Memoria de trabajo & Subtest de Dígitos (WAIS) &, 89 &, 80 \\
\hline
\end{tabular}


el deterioro temprano de funciones vinculadas a la memoria. Sin embargo, su aporte diagnóstico se encontraría principalmente en la comparación entre sujetos con envejecimiento normal y demencia temprana ${ }^{77}$.

\section{Conclusión}

Para la detección temprana de la EA se han propuesto biomarcadores y pruebas neuropsicológicas; los primeros buscan identificar huellas fisiopatológicas de la enfermedad, mientras que las segundas buscan determinar el perfil neuropsicológico del paciente para que el clínico contraste esta información con lo que se espera encontrar en el curso de la EA.

Se han estudiado numerosos biomarcadores; actualmente estudios que integran distintos parámetros biológicos han mostrado mayor sensibilidad y especificidad que los mismos parámetros usados de manera aislada.

También se han propuesto numerosas pruebas neuropsicológicas para evaluar las distintas funciones cognitivas en la fase temprana de la EA. Sin embargo, es necesario contar con datos claros sobre confiabilidad, validez y sensibilidad de las pruebas útiles para esta tarea. Lamentablemente, aún gran parte de las versiones de estas pruebas usadas en Chile son extranjeras sin datos normativos claros para población chilena.

\section{Referencias}

1. Valls-Pedret C, Molinuevo, J, Rami L. Diagnóstico precoz de la enfermedad de Alzheimer. Rev Neurol 2010; 51: 471-80.

2. Campion D, Dumanchin C, Hannequin D, Dubois B, Belliard S, Puel M, et al. Early onset autosomal dominant Alzheimer disease: prevalence, genetic heterogeneity and mutation spectrum. Am J Hum Genet 1999; 65: 664-70.

3. Molinuevo J, González R, Plarrumaní A. Demencias: entidades nosológicas y sintomatología neuropsicológica. En: Peña-Casanova. Neurología de la conducta y neuropsicología. España: Médica Panamericana 2007; 363-79.

4. American Psychiatric Association. Manual Diagnóstico y Estadístico de los Trastornos Mentales. Barcelona: Masson, 2002.
5. Flicker C, Ferris SH, Reisberg B. Mild cognitive impairment in the elderly: predictors of dementia. Neurology 1991; 41: 1006-9.

6. Petersen RC, Smith GE, Waring SC, Ivnik RJ, Tangalos EG, Kommen E. Mild cognitive impairment: clinical characterization and outcome. Arch Neurol 1999; 56: 303-8.

7. Ritchie K. Mild cognitive impairment an epidemiological perspective. Dialogues Clin Neurosci 2004; 6: 401-8.

8. Sarazin M, Berr C, De Rotrou J, Fabrigoule C, Pasquier F, Legrain S, et al. Amnestic syndrome of the medial temporal type identifies prodromal AD: a longitudinal study. Neurology 2007; 69: 1859-67.

9. Bouwman FH, Verwey NA, Klein M, Kok A, Blankenstein MA, Sluimer JD, et al. New research criteria for the diagnosis of Alzheimer's disease applied in a memory clinic population. Dement Geriatr Cogn Disord 2010; 30: 1-7.

10. Frisoni GB, Galluzzi S, Signorini M, Garibotto V, Paghera B, Binetti G, et al. Preliminary evidence of validity of the revised criteria for Alzheimer disease diagnosis: report of 2 cases. Alzheimer Dis Assoc Disord 2010; 24: 108-14.

11. Tounsi H, Deweer B, Ergis AM, Van der Linden M, Pillon B, Michon A, et al. Sensitivity to semantic cuing: an index of episodic memory dysfunction in early Alzheimer disease. Alzheimer Dis Assoc Disord 1999; 13: 38-46.

12. Monge-Argilés JA, Sánchez-Payá J, Muñoz-Ruiz C, Pampliega-Pérez A, Montoya-Gutiérrez J, Leiva-Santana C. Biomarcadores en el líquido cefalorraquídeo de pacientes con deterioro cognitivo leve: metaanálisis de su capacidad predictiva para el diagnóstico de la enfermedad de Alzheimer. Rev Neurol 2010; 50: 193-200.

13. Ittner L, Götz J. Amyloid-and tau-a toxic pas de deux in Alzheimer's disease. Nat Rev Neurosci 2010; 12: 67-72.

14. Seeman P, Seeman N. Alzheimer's disease: -amyloid plaque formation in human brain. Synapse 2011; 65: 1289-97.

15. LaFerla F, Green K, Oddo S. Intracellular amyloid- in Alzheimer's disease. Nat Rev Neurosci 2007; 8: 499-509.

16. Oddo S, Vasilevko V, Caccamo A, Kitazawa A, Cribbs D, LaFerla F. Reduction of soluble Abeta and tau, but not soluble Abeta alone, ameliorates cognitive decline in transgenic mice with plaques and tangles. J Biol Chem 2006; 281: 39413-23.

17. Kawahara M. Neurotoxicity of -amyloid protein: oligomerization, channel formation, and calcium dyshomeostasis. Curr Pharm Des 2010; 16: 2779-89.

18. Braak H, Braak E. Neuropathological staging of Alzheimer-related changes. ActaNeuropathol 1991; 82: 239-59. 
19. Guimerà A, Gironès X, Cruz-Sánchez F. Actualización sobre la patología de la enfermedad de Alzheimer. Rev Esp Patol 2001; 35: 21-48.

20. Kagan B, Thundimadathil J. Amyloid peptide pores and the beta sheet conformation. Adv Exp Med Biol 2010; 677: 150-67.

21. Biomarkers Definitions Working Group. Biomarkers and surrogate endpoints: preferred definitions and conceptual framework. Clin Pharmacol Ther 2001; 69: 8995.

22. Perrin RJ, Fagan AM, Holtzman DM. Multimodal techniques for diagnosis and prognosis of Alzheimer's disease. Nature 2009; 461: 916-22.

23. Blennow K, Hampel H, Weiner M, Zetterberg H. Cerebrospinal fluid and plasma biomarkers in Alzheimer disease. Nat Rev Neurol 2010; 6: 131-44.

24. Ashburner J. and K. Friston. 2000. Voxel-Based Morphometry-The Methods. NeuroImage 2000; 11: 805-21.

25. Molinuevo J, Gómez-Anson B, Monte G, Bosch B, Sánchez-Valle R, Rami L. Neuropsychological profile of prodromal Alzheimer's disease (Prd-AD) and their radiological correlates. Adv Exp Med Biol 2011; 677: 150-67.

26. Grady CL, Furey ML, Pietrini P, Horwitz B, Rapoport SI. Altered brain functional connectivity and impared short-term memory in Alzheimer's disease. Brain 2001; 124: 739-56.

27. Mintun MA, Larossa GN, Sheline YI, Dence CS, Lee SY, Mach RH, et al. [11C] PIB in a nondemented population: potential antecedent marker of Alzheimer disease. Neurology 2006; 67: 446-52.

28. Blennow K. Cerebrospinal fluid protein biomarkers for Alzheimer's disease. NeuroRx 2004; 1: 21325.

29. Fagan AM, Roe CM, Xiong C, Mintun MA, Morris JC, Holtzman DM. Cerebrospinal fluid tau/ -amyloid 42 ratio as a prediction of cognitive decline in nondemented older adults. Arch Neurol 2007; 64: 343-9.

30. Hampel H, Teipel SJ, Fuchsberger T, Andreasen N, Wiltfang $J$, Otto $M$, et al. Value of CSF betaamyloid1-42 and tau as predictors of Alzheimer's disease in patients with mild cognitive impairment. Mol Psychiatry 2004; 7: 705-10.

31. Lewczuk P, Esselmann H, Otto M, Maler JM, Henkel AW, Eikenberg $\mathrm{O}$, et al. Neurochemical diagnosis of Alzheimer's dementia by CSF Abeta42, Abeta42/Abeta40 ratio and total tau. Neurobiol Aging 2004; 25: 273-81.

32. Fagan AM, Mintun MA, Mach RH, Lee SY, Dence CS, Shah AR, et al. Inverse relation between in vivo amyloid imaging load and cerebrospinal fluid Abeta42 in humans. Ann Neurol 2006; 59: 512-9.
33. Hansson $\mathrm{O}$, Zetterberg $\mathrm{H}$, Buchhave $\mathrm{P}$, Andreasson U, Londos E, Minthon L, et al. Prediction of Alzheimer's disease using the CSF Abeta42/Abeta40 ratio in patients with mild cognitive impairment. Dement Geriatr Cogn Disord 2007; 23: 316-20.

34. Lewczuk P, Esselmann H, Meyer M, Wollscheid V, Newmann M, Otto M, et al. The amyloid-beta (Abeta) peptide pattern in cerebrospinal fluid in Alzheimer's disease: evidence of a novel carboxyterminally elongated Abeta peptide. Rapid Commun Mass Spectrom 2003; 17: 1291-6.

35. Iqbal K, Alonso AC, Gong CX, Khatoon S, Pei JJ, Wang JZ, et al. Mechanisms of neurofibrillary degeneration and the formation of neurofibrillary tangles. J Neural Transm Suppl 1998; 53: 169-80.

36. Formichi P, Battisti C, Radi E, Federico A. Cerebrospinal fluid tau, A, and phosphorylated tau protein for the diagnosis of Alzheimer's disease. J Cell Physiol 2006; 208: 39-46.

37. Hampel H, Buerger K, Zinkowski R, Teipel SJ, Goernitz A, Andreasen N, et al. Measurement of phosphorylated tau epitopes in the differential diagnosis of Alzheimer disease: a comparative cerebrospinal fluid study. Arch Gen Psychiatry 2004; 61: 95-102.

38. Koopman K, Le Bastard N, Martin JJ, Nagels G, De Deyn PP, Engelborghs S. Improved discrimination of autopsy-confirmed Alzheimer's disease (AD) from non-AD dementias using CSF P-tau181P. Neurochem Int 2009; 55: 214-8.

39. Buerger K, Teipel S, Zinkowski R, Blennow K, Arai H, Engel R. CSF tau protein phosphorylated at threonine 231 correlates with cognitive decline in MCI subjects. Neurology 2002; 59: 6279.

40. Mayeux R, Saunders AM, Shea S, Mirra S, Evans D, Roses $\mathrm{AD}$, et al. Utility of the Apolipoprotein E genotype in the diagnosis of Alzheimer's disease. N Engl J Med 1998; 338: 506-11.

41. Growdon J. Biomarkers of Alzheimer disease. Arch Neurol 1999; 56: 2813.

42. Pratico D, Sung S. Lipid peroxidation and oxidative imbalance: early functional events in Alzheimer's disease. J Alzheimers Dis 2004; 6: 1715.

43. Pratico D, Clark C, Lee V, Trojanowski J, Rokach J, Fitzgerald G. Increased 8,12isoiPF2alphaVI in Alzheimer's disease: correlation of a noninvasive index of lipid peroxidation with disease severity. Ann Neurol 2000; 48: 80912.

44. Thal L, Kantarci K, Reiman E, Klunk W, Weiner M, Zetterberg $\mathrm{H}$. The role of biomarkers in clinical trials for Alzheimer disease. Alzheimer Dis Assoc Disord 2006; 20: 615. 
45. Lee JM, Blennow K, Andreasen N, Laterza O, Modur $\mathrm{V}$, Modur V, et al. The brain injury biomarker VLP-1 is increased in the cerebrospinal fluid of Alzheimer disease patients. Clin Chem 2008; 54: 1617-23.

46. Craig-Shapiro R, Fagan AM, Holtzman DM. Biomarkers of Alzheimer's disease. Neurobiol Dis 2009; 35: 39-46.

47. Maddalena A, Papassotiropoulos A, Müller-Tillmanns B, Jung HH, Heigi T, Nitsch RM, et al. Biochemical diagnosis of Alzheimer disease by measuring the cerebrospinal fluid ratio of phosphorylated tau protein to beta-amyloid pptide 42. Arch Neurol 2003; 60: 1202-6.

48. Moulin CJ, Laine M, Rinne JO, Kaasinen V, Sipilä H, Hiltunen J, et al. Brain function during multi-trial learning in mild cognitive impairment: a PET activation study. Brain Res 2007; 1136: 132-41.

49. Ray S, Britschgi M, Herbert C, Takeda-Uchimura Y, Boxer A, Blennow $\mathrm{K}$, et al. Classification and prediction of clinical Alzheimer's diagnosis based on plasma signaling proteins. Nat Med 2007; 13: 1359-62.

50. O’Bryant SE, Xiao G, Barber R, Reisch J, Doody R, Fairchild $\mathrm{T}$, et al. A serum protein-based algorithm for the detection of Alzheimer disease. Arch Neurol 2010; 67: 1077-81.

51. Raichle ME, MacLeod AM, Snyder AZ, Powers WJ, Gusnard DA, Shulman GL. A default mode of brain function. Proc NatlAcadSci U S A 2001; 98: 676-82.

52. Fair DA, Cohen AL, Dosenbach NU, Church JA, Miezin FM, Barch DM, et al. The maturing architecture of the brain's default network. Proc Natl Acad Sci U S A 2008; 105: 4028-32.

53. Fox MD, Snyder AZ, Vincent JL, Corbetta M, Van Essen DC, Raichle ME. The human brain is intrinsically organized into dynamic, anticorrelated functional networks. Proc Natl Acad Sci U S A 2005; 102: 9673-8.

54. Greicius MD, Krasnow B, Reiss AL, Menon V. Functional connectivity in the resting brain: a network analysis of the default mode hypothesis. Proc Natl Acad Sci USA 2003; 100: 253-8.

55. Buckner RL, Andrews-Hanna JR, Schacter DL. The brain's default network: anatomy, function, and relevance to disease. Ann NY Acad Sci 2008; 1124: 1-38.

56. Damoiseaux JS, Prater KE, Miller BL, Greicius MD. Functional connectivity tracks clinical deterioration in Alzheimer's disease. Neurobiol Aging 2011; In Pess: doi:10.1016/j.neurobiolaging.2011.06.024

57. Sorg C, Riedl V, Mühlau M, Calhoun VD, Eichele T, Läer L, et al. Selective changes of resting-state networks in individuals at risk for Alzheimer's disease. Proc Natl Acad Sci USA 2007; 104: 18760-5.

58. Gili T, Cercgnani M, Serra L, Perri R, Giove F, Maraviglia $\mathrm{B}$, et al. Regional brain atrophy and functional disconnection across Alzheimer's disease evolution. J Neurol Neurosurg Psychiatry 2011; 82: 58-66.

59. Petrella JR, Sheldon FC, Prince SE, Calhoun VD, Doraiswamy PM. Default mode network connectivity in stable vs progressive mild cognitive impairment. Neurology 2011; 76: 511-7.

60. Bero AW, Yan P, Roh JH, Cirrito JR, Stewart FR, Raichle ME, et al. Neuronal activity regulates the regional vulnerability to amyloid-deposition. Nat Neurosci 2011; 14: 750-6.

61. Buckner RL, Snyder AZ, Shannon BJ, LaRossa G, Sachs $\mathrm{R}$, Fotenos AF, et al. Molecular, structural and functional characterization of Alzheimer's disease: evidence for a relationship between default activity, amyloid, and memory. J Neurosci 2005; 25: 7709-17.

62. Buckner RRL, Sepulcre J, Talukdar T, Krienen FM, Liu $\mathrm{H}$, Hedden $\mathrm{T}$, et al. Cortical hubs revealed by intrinsic functional connectivity: mapping, assessment of stability, and relation to Alzheimer's disease. J Neurosci 2009; 29: 1860-73.

63. Peña-Casanova J. Test Barcelona Revisado. Normalidad, semiología y patologías neuropsicológicas. Barcelona: Masson; 2005.

64. Peña-Casanova J. Gramunt N, Gich J. Tests neuropsicológicos. Fundamentos para una neuropsicología clínica basada en evidencias. Barcelona: Masson; 2004.

65. Peña-Casanova J. Exploración neuropsicológica de la enfermedad de Alzheimer. En: Alberca R, Pousa S., 3 ed. Enfermedad de Alzheimer y otras demencias; 2006. P. 47-73.

66. Strub RL, Black FW. The mental status examination in neurology. Philadelphia: FA Davis Company; 1985.

67. Trzepacz PT, Baker R. The psychiatric mental status examination. Oxford: Oxford University Press; 1993.

68. Folstein MF, Folstein SE, McHugh PR. "Mini-mental state". A practical method for grading the cognitive state of patients for the clinicians. J Psychiatr Res 1975; 12: 189-98.

69. Böhm P, Peña-Casanova J, Gramunt N, Manero R, Terrón C, Quiñones S. Spanish version of the Memory Impairment Screen (MIS): normative data and discriminant validity. Neurología 2005; 20: 402-11.

70. Parsey C, Schmitter-Edgecombe M. Quantitative and qualitative analyses of the clock drawing test in mild cognitive impairment and Alzheimer disease: evaluation of a modified scoring system. J Geriatr Psychiatry Neurol 2011; 24: 108-18.

71. Brown J, Pengas G, Dawson K, Brown LA, Clatworthy P. Self administered cognitive screening tests (TYM) for detection of Alzheimer's disease: cross sectional study. Br Med J 2009; 338: b2030. 
Evaluación Alzheimer en etapa temprana: biomarcadores y tests neuropsicológicos - R. Lanfranco et al

72. Mohs RC, Rosen WG, Davis KL. The Alzheimer's disease assessment scale: and instrument for assessing treatment efficacy. Psychofarmacol Bull 1983; 19: 448-50.

73. Roth M, Huppert FA, Tym E, et al. CAMDEX: The Cambridge examination for mental disorders for the elderly. Cambridge: Cambridge University Press; 1988.

74. Cole M, Dastoor D. The Hierarchical Dementia Rating Scale. J Clin Exper Gerontol 1983; 5: 219-34.

75. Nasreddine Z, Phillips N, Bédirian V, Charboneau S, Whitehead V, Collin I, et al. The Montreal Cognitive Assessment, MoCA: A brief screening tool for Mild Cognitive Impairment. J Am Geriatr Soc 2005; 53: 695-9.
76. Allegri R, Harris P, Drake M. La evaluación neuropsicológica en la enfermedad de Alzheimer. Rev Neurol Arg 2000; 25 (supl 1): 11-5.

77. Salmon DP, Hodges JR. Neuropsychological assessment of early onset dementia. En: Hodges JR, ed. Early-onset dementia. Oxford: Oxford University Press; 2001. P. 4773.

78. Benito-Cuadrado MM, Esteba-Castillo S, Böhm P, Cejudo-Bolívar J, Peña-Casanova J. Semantic verbal fluency of animals; a normative and predictive study in a spanish population. J Clin Exp Neuropsychol 2002; 24: 1117-22. 